\title{
Estudio de la adquisición de subcompetencias sobre comunicación audiovisual de los maestros en formación inicial
}

\author{
Antonio de Pro Bueno ${ }^{1}$, Carlos de Pro Chereguini ${ }^{1}$ y Javier Rodríguez Moreno ${ }^{2}$ \\ ${ }^{1}$ Dpto. Didáctica de las Ciencias Experimentales. Universidad de Murcia. \\ ${ }^{2}$ Dpto. Didáctica y Organización Escolar. Universidad de Jaén.
}

[Recibido el 25 de octubre de 2018, aceptado el 28 de diciembre de 2018]

El trabajo tiene como objetivo explorar cómo utilizan los alumnos del Grado de Educación Primaria sus conocimientos ante unos anuncios publicitarios relacionados con el ahorro energético. Se han analizado las respuestas de unos estudiantes, desde la perspectiva de los conocimientos científicos y la adquisición de unas subcompetencias sobre comunicación audiovisual. Al realizarse antes de cualquier intervención sobre Didáctica de las Ciencias Experimentales, los resultados nos plantean retos sobre el contenido objeto de enseñanza en la formación inicial de maestros en el ámbito de la Didáctica de las Ciencias Experimentales.

Palabras clave: formación inicial de maestros; conocimientos científicos; adquisición de competencias; ahorro energético; spot publicitario.

\section{Study of the acquisition of subcompetences on audiovisual communication by teachers in initial training}

The objective of the work is to explore how the students of the Primary Education Degree use their knowledge in front of advertisements related to energy saving. We analyze the answers of some students, from the perspective of scientific knowledge and the acquisition of some subcompetences on audiovisual communication. When carried out before any intervention on Didactics of Experimental Sciences, the results present challenges to us on the subject matter of teaching in the initial formation of teachers in the field of Experimental Sciences Didactics.

Keywords: initial teacher training; scientific knowledge; acquisition of skills; energy saving; advertising spot.

Para citar el artículo. De Pro Bueno, A., De Pro Chereguini, C. y Rodríguez Moreno, J. (2019). Estudio de la adquisición de subcompetencias sobre comunicación audiovisual de los maestros en formación inicial. Ápice. Revista de Educación Científica, 3(1), 29-39. DOI: https://doi.org/10.17979/ arec.2019.3.1.4618

Contacto.nono@um.es,cpro@um.es,jrmoreno@ujaen.es 


\section{Introducción}

Si revisamos los trabajos que se recogen en los últimos números de las revistas del ámbito de la Didáctica de las Ciencias Experimentales (DCE) o asistimos a los eventos -congresos, jornadas, encuentros...- más conocidos de este campo de conocimiento, se puede afirmar que el crecimiento, como área, es bastante evidente y significativo (Perales, 2018). Sin embargo, a pesar de la cantidad y calidad de las contribuciones, observamos con preocupación que no se aprecian cambios multitudinarios o generalizados en las aulas. ¿’Por qué no llegan los logros de la investigación o de la innovación a las clases habituales de Ciencias? Más allá de otros factores que sin duda inciden en la situación, uno de ellos podría ser el desconocimiento de las mismas por los docentes.

En nuestro contexto educativo, la estrategia de cambio utilizada institucionalmente, desde hace algún tiempo, ha sido la "reforma de los planes de estudios". Pero, han sido tantos "los remedios que auguraban el final de los problemas" que, lejos de ser una herramienta para introducir mejoras, la proliferación de reformas ha generado confusión, confrontación y, sobre todo, desencanto. Desde nuestra perspectiva, creemos que, si queremos cambiar la enseñanza de las Ciencias, es una condición necesaria cambiar la formación del profesorado que la enseña. Y, además, debe hacerse en todas las etapas del desarrollo profesional: en la formación inicial, en el acceso a la profesión docente, en los inicios como profesorado novel, etc.

Ahora bien, para plantear una alternativa a los planes y acciones formativas existentes creemos que hay que conocer las características de aquellos a los que va dirigida la formación, tener claras las necesidades de los que forman parte del sistema educativo, definir el perfil profesional del docente que queremos, estudiar lo que funciona y lo que hay que mejorar de lo que hacemos, tener en cuenta las aportaciones de la investigación e innovación que recoge la literatura especializada... Las Administraciones educativas se han acostumbrado a "ser originales" $y$, sin tener datos sobre lo que hacemos razonablemente bien y lo que es manifiestamente mejorable, se hacen propuestas que no sólo no resuelven los problemas, sino que muchas veces complican más su resolución.

También hay que considerar que el desarrollo profesional de los docentes se debe realizar a lo largo de su vida y, por tanto, hay que tener claras qué competencias queremos desarrollar en su formación inicial y cuáles deben dejarse para más adelante. Ni se puede repetir formación. Ni se debe tratar todas las facetas del ejercicio profesional en la primera etapa como maestros.

\section{Planteamiento de los problemas de investigación}

Nuestro trabajo se sitúa en la realidad que tenemos en la formación inicial de maestros de Educación Primaria; concretamente en el ámbito de la enseñanza de las Ciencias. Tenemos unas directrices oficiales en el Grado de Educación Primaria, se ha ampliado la duración de la titulación, se ha introducido el tema de las competencias, se han elaborado unos planes de estudios (por cierto, bastante dispares a pesar de las directrices...), se han revisado los currículos de los niveles no universitarios y las pruebas de acceso a la universidad...

Pero, en este nuevo contexto, ¿cómo llegan nuestros futuros maestros para afrontar su formación en DCE? ¿tienen una formación científica adecuada? Tradicionalmente nuestras materias no están, en general, en primer curso, ¿̇han mejorado sus conocimientos psicopedagógicos adquiridos en los cursos anteriores del Grado? Si ya han realizado algún periodo de Prácticas, ¿̇ha incidido sus experiencias en una visión más acertada de lo que es la práctica profesional? 
Creemos que la formación inicial de los maestros es una etapa clave en su desarrollo como docentes. Sin embargo, aunque formalmente nuestros títulos de Grado de Educación Primaria están acreditados y verificados, tenemos la sensación, como participantes en el mismo, de que han mejorado determinados aspectos pero que persisten otros que ya habíamos detectado como problemáticos.

Así, a menudo planteamos a nuestros alumnos que deben contemplar, en la selección de contenidos, temáticas que atiendan sus necesidades como ciudadanos, que contemplen experiencias y situaciones cotidianas en las que los estudiantes tengan que utilizarlos, que acerquen la "ciencia de la calle" al aula donde se enseñen las ciencias, que permitan generar una actitud crítica hacia el uso que se hace de los descubrimientos científicos, etc. Pero ¿sabemos si ellos tienen conocimientos y han adquirido las competencias necesarias para poder enseñarlos?

Necesitamos conocer qué saben y cómo utilizan los estudiantes del Grado de Educación Primaria sus conocimientos. Como puede verse, la problemática es compleja y probablemente sea imposible hacer frente a todos los interrogantes que se derivan de ella en un solo trabajo. Por ello, nos hemos centrado en algo concreto. Teniendo en cuenta todo lo reseñado, nos hemos planteado los siguientes Problemas de Investigación:

- Problema Principal Uno (PP1): ¿Tienen conocimientos los futuros maestros para comprender e interpretar un anuncio televisivo sobre Ahorro energético?

- Problema Principal Dos (PP2): ¿Cuál es el grado de desarrollo de subcompetencias relacionadas con la comunicación audiovisual ante un anuncio televisivo sobre Ahorro energético?

\section{Revisión de antecedentes}

Este trabajo pretende valorar, previamente a nuestra intervención en DCE, algunos conocimientos -científicos y didácticos- y el desarrollo de una competencia de un grupo de futuros profesores ante spots publicitarios sobre el ahorro energético.

Para llevar adelante nuestro estudio, hemos de considerar los problemas habituales que tienen los estudiantes sobre el aprendizaje de la energía, los logros que hayan tenido en su formación psicopedagógica y las subcompetencias que se pueden poner en juego cuando se utiliza un audiovisual (AV) de estas características.

En la Tabla 1 se recoge una síntesis de los problemas de aprendizaje que presentan los estudiantes frente a la temática sobre Energía (Pro, 2014); obviamente no todas pueden encontrarse en las noticias de prensa, campañas publicitarias, programas de televisión, etc. Aunque están referidas a niveles anteriores del sistema educativo, es posible que algunas permanecieran en éste, ya que la exploración se realizó antes de impartir las materias de DCE que se contemplan en el plan de estudios del Grado de Educación Primaria.

Por otro lado, no podemos olvidar que la enseñanza en el siglo XXI debe tener, entre sus prioridades, traer lo que hay en la calle a las aulas. En este sentido, sabemos que la publicidad juega un papel fundamental en la educación fuera de la escuela y, por lo tanto, resulta obligada su intención, su análisis, su valoración... por parte de los ciudadanos. Para ello, es necesario que el futuro maestro sea capaz de plantear objetivos y, en base a estos, seleccionar contenidos y proponer actividades que permitan que los niños los alcancen.

Los estudiantes que participan en nuestra experiencia habían recibido formación psicopedagógica en los cursos anteriores del Grado. No obstante, en la Tabla 2 se recogen dificultades encontradas en otros trabajos cuando hablamos de conocimientos didácticos y diseño de actividades (Pro y Saura, 2000; 2007; Pro, Saura y Sánchez, 2000; Mellado, 
Tabla 1. Dificultades en torno a la energía

\section{PROBLEMAS DE APRENDIZAJE (Energía)}

- Consideran que la energía es "algo" -una especie de sustancia- que puede almacenarse, trasportarse, darse, quitarse...; incluso, que está en el interior de los cuerpos y de los objetos.

- Tienen confusiones terminológicas por el uso de muchos términos en el lenguaje cotidiano (trabajo-esfuerzo-energía-potencia) o por la influencia de la publicidad o del cine (bola de energía, producto bioenergético, alimentos sin energía, energía de la vida...).

- Asocian el concepto de energía al movimiento, a la actividad, a la vitalidad de los seres vivos..

- Les cuesta comprender el principio de conservación; les es más intuitivo el de degradación ("cuando se ha utilizado una energía, no se puede recuperar para usarla de nuevo").

- Por motivos académicos utilizan a veces expresiones no adecuadas: la energía es la capacidad para producir trabajo, el calor es una forma de energía...; no suele ser muy preocupante porque no comprenden el alcance de lo que han memorizado.

- La mayoría son conscientes de que los problemas de los recursos energéticos les afectan (sube el precio de la gasolina, el del trasporte y el de otros productos) pero no tienen la sensación que la producción energética les afecte ni incidan en la conservación del medio "cuanta más energía se produzca, mejor", "si necesitamos más energía, construyamos más centrales" ...

- Tienen ideas distorsionadas de las fuentes alternativas: "cuando se construye un parque eólico, aumenta el viento en la zona", "si se coloca una placa fotovoltaica, atrae el calor" ...

- No reconocen las ideas fundamentales, contradictorias, semejantes... en un material escrito pero el problema se incrementa cuando se usa la prensa diaria o información de internet; necesitan una secuencia dirigida de cuestiones concretas para trabajar.

Tabla 2. Dificultades respecto a algunos conocimientos didácticos

\section{PROBLEMAS DE APRENDIZAJE (Conocimientos didácticos)}

- Consideran que el currículum oficial es lo que marcan los libros de texto.

- Convierten cualquier proceso de planificación en una sucesión técnica de los elementos (objetivos, contenidos, actividades...), pero no siempre se preocupan de la coherencia entre ellos.

- Utilizan etiquetas (constructivismo, competencias, aprendizaje significativo, indagación, ABP, evaluación formativa...) que no son capaces de concretar en acciones y actividades concretas.

- No son capaces de incluir los tres tipos de contenidos; predominan los conceptuales.

- Conocen las características psicoevolutivas, pero muchos las utilizan sólo como limitaciones del aprendizaje, para "infantilizar" el currículum.

- Cuando conocen las ideas previas, son capaces de detectarlas, pero no las utilizan en el proceso de construcción del aprendizaje.

- Tienen la creencia que la inclusión de un recurso (el trabajo de laboratorio, las TICs, las salidas del aula, el trabajo en grupo...) es suficiente para mejorar los resultados de aprendizaje.

- Tienen dificultades para establecer secuencias, actividades, contenidos... con diferentes niveles de complejidad e intenciones educativas.

- Confunden entretener con aprender.

- Siguen considerando la evaluación como elemento de control, centrado en el éxito académico del alumnado, asociado al rendimiento individual, al uso de pruebas... 
2001; van Driel, Beijaard y Verloop, 2001; Jarvis y Pell, 2004; Gairín, 2009; Marcelo, 2011; Pro y Pro, 2012; Opre, 2013; Grangeat y Kapelari, 2015; Bravo y Cofre, 2016; Pro, Pro y Serrano, 2018).

En cuanto a las subcompetencias explícitas en el uso del recurso audiovisual, teniendo en cuenta otros trabajos (Pérez, 2009; Jiménez, 2010; Pedrinaci, 2012; Pro, Pro y Serrano, $2017 ; 2018)$, hemos utilizado las que se recogen en la Tabla 3.

Tabla 3. Subcompetencias de la comprensión audiovisual

\begin{tabular}{|l|l|}
\hline Procesos cognitivos & Subcompetencias \\
\hline $\begin{array}{l}\text { Localizar y obtener } \\
\text { información }\end{array}$ & $\begin{array}{l}\text { - Identificación de ideas en el AV (en la imagen o en el audio). } \\
\text { - Búsqueda de información relacionada con el AV }\end{array}$ \\
\hline $\begin{array}{l}\text { Interpretar y utilizar } \\
\text { información }\end{array}$ & $\begin{array}{l}\text { - Significado de términos, expresiones, fragmentos... en el AV (en la } \\
\text { imagen o en el audio). } \\
\text { audio). }\end{array}$ \\
\hline $\begin{array}{l}\text { Reflexionar o valorar } \\
\text { información }\end{array}$ & $\begin{array}{l}\text { - Localización de errores en el AV (en la imagen o en el audio). } \\
\text { imagen o en el audio). } \\
\text { - Realización de inferencias lejanas al AV. }\end{array}$ \\
\hline $\begin{array}{l}\text { Comprender } \\
\text { globalmente }\end{array}$ & $\begin{array}{l}\text { - Identificación de ideas principales del AV. } \\
\text { - Realización de un resumen del AV. }\end{array}$ \\
\hline
\end{tabular}

\section{Marco empírico}

El diseño utilizado es de tipo exploratorio. El grupo de participantes estuvo constituido por dos grupos de 3o del Grado de Educación Primaria; había 127 matriculados, pero, por motivos ajenos a la investigación, sólo tenemos la información de 99.

Eran 75 mujeres y 24 hombres. Sólo un 30\% había cursado estudios de carácter científico en su etapa anterior del sistema educativo (Bachillerato o Formación Profesional). Al realizar el estudio antes de abordar cualquier materia de Didáctica de las Ciencias Experimentales (DCE), habían pasado dos años, como mínimo, desde la última vez que estudiaron Ciencias u otras materias afines. Todos habían cursado materias psico-pedagógicas; entre ellas, Planificación de la Acción Educativa, Medios materiales y TIC y Psicología del Desarrollo.

El instrumento de recogida de información era una composición de cuatro spots publicitarios promovidos por el Instituto para la Diversificación y Ahorro de la Energía (IDAE) para sensibilizar a la ciudadanía sobre el ahorro energético (https://www.youtube.com/watch?v=LRmMVxtjZuk). Los protagonistas eran jugadores de fútbol de la Selección Española, personajes conocidos por todos los estudiantes, y su presencia había sido habitual en las diversas cadenas televisivas. El AV duraba algo menos de 1 minuto.

El entrevistador se situaba delante de los estudiantes y les reclamaba su atención: "Estad atentos. Sólo voy a hacerlo dos veces" (así lo hicimos con una separación de 3 minutos). En la Figura 1, se recogen algunas escenas.

Una vez visualizado el video, el profesor les pasó un cuestionario con las preguntas que se recogen en la Tabla 4; se señalan las subcompetencias correspondientes. 


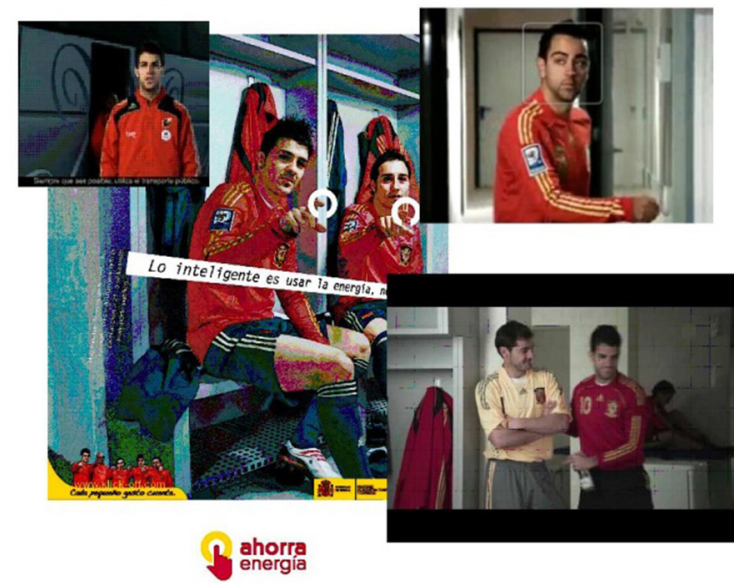

Figura 1. Escenas de los anuncios publicitarios

Tabla 4. Conocimientos y subcompetencias de las cuestiones planteadas

\begin{tabular}{|c|c|c|}
\hline Preguntas & Conocimientos & Subcompetencias \\
\hline 1. ¿Qué ha dicho Casillas? & $\begin{array}{l}\text { Ahorro energético. Consumo } \\
\text { energético. }\end{array}$ & $\begin{array}{l}\text { Identificación de } \\
\text { ideas en AV }\end{array}$ \\
\hline $\begin{array}{l}\text { 2. ¿Qué quiere decir que un electrodo- } \\
\text { méstico es de clase } A \text { ? }\end{array}$ & Consumo energético. Clase $A$. & $\begin{array}{l}\text { Significación de tér- } \\
\text { minos del AV }\end{array}$ \\
\hline $\begin{array}{l}\text { 3. ¿Crees que el uso del trasporte pú- } \\
\text { blico puede ayudar a ahorrar energía? } \\
\text { Justifícalo. }\end{array}$ & $\begin{array}{l}\text { Ahorro energético. Gasoil. } \\
\text { Consumo energético. }\end{array}$ & $\begin{array}{l}\text { Posicionamiento } \\
\text { argumentado ante } \\
\text { un AV }\end{array}$ \\
\hline $\begin{array}{l}\text { 4. ¿Qué ha hecho Cazorla al ser susti- } \\
\text { tuido? }\end{array}$ & $\begin{array}{l}\text { Circuito eléctrico. Interruptor. } \\
\text { Corriente eléctrica. }\end{array}$ & $\begin{array}{l}\text { Identificación de } \\
\text { ideas en AV }\end{array}$ \\
\hline 5. ¿Por qué lo ha hecho? & $\begin{array}{l}\text { Ahorro energético. Energía } \\
\text { eléctrica. }\end{array}$ & $\begin{array}{l}\text { Inferencia cercana } \\
\text { al AV }\end{array}$ \\
\hline $\begin{array}{l}\text { 6. ¿Qué se dice sobre la T del aire } \\
\text { acondicionado? }\end{array}$ & $\begin{array}{l}\text { Aire acondicionado. Tempera- } \\
\text { tura. }\end{array}$ & $\begin{array}{l}\text { Identificación de } \\
\text { ideas en AV }\end{array}$ \\
\hline 7. ¿Por qué lo ha dicho? & $\begin{array}{l}\text { Ahorro energético. Consumo } \\
\text { energético. }\end{array}$ & $\begin{array}{l}\text { Inferencia cercana } \\
\text { al AV }\end{array}$ \\
\hline 8. ¿Qué es un termostato? & Termostato. Temperatura. & $\begin{array}{l}\text { Significación de tér- } \\
\text { minos del AV }\end{array}$ \\
\hline $\begin{array}{l}\text { 9. ¿Qué consideras más barato el uso } \\
\text { de climatizadores eléctricos o de estu- } \\
\text { fas de gas? }\end{array}$ & $\begin{array}{l}\text { Climatizador. Estufa. Energía } \\
\text { eléctrica. Gas. Consumo ener- } \\
\text { gético. Ahorro energético. }\end{array}$ & $\begin{array}{l}\text { Posicionamiento } \\
\text { argumentado ante } \\
\text { un AV }\end{array}$ \\
\hline $\begin{array}{l}\text { 10. Di las tres ideas más importantes } \\
\text { del anuncio que has visto }\end{array}$ & $\begin{array}{l}\text { Ahorro energético. Consumo } \\
\text { energético }\end{array}$ & $\begin{array}{l}\text { Resumen ideas prin- } \\
\text { cipales del AV }\end{array}$ \\
\hline $\begin{array}{l}\text { 11. Diseña una actividad de enseñanza } \\
\text { para sensibilizar al alumnado de } 2 \text { o ci- } \\
\text { clo de Educación Primaria sobre la im- } \\
\text { portancia del ahorro energético. Utiliza } \\
\text { uno de los cuatro spots del video. }\end{array}$ & $\begin{array}{l}\text { Ahorro energético. Consumo } \\
\text { energético. Importancia ener- } \\
\text { gía en la vida cotidiana. Desa- } \\
\text { rrollo sostenible. } \\
\text { Diseño actividad AV. }\end{array}$ & $\begin{array}{l}\text { Inferencia cercana } \\
\text { al AV }\end{array}$ \\
\hline $\begin{array}{l}\text { 12. Si tuvieras que buscar información } \\
\text { en internet sobre ahorro energético, } \\
\text { ¿qué harías? }\end{array}$ & Ahorro energético. & $\begin{array}{l}\text { Búsqueda de infor- } \\
\text { mación a partir de } \\
\text { un AV }\end{array}$ \\
\hline
\end{tabular}


Los estudiantes contestaron por escrito. Luego identificamos y analizamos cada unidad de información facilitada por los que respondieron dicho cuestionario.

\section{Resultados}

\section{En relación con el PP1}

Como dijimos hay cuestiones de carácter cientifico; respecto a ellas, podemos decir:

- Se produjeron muchas confusiones terminológicas: electricidad-corriente eléctrica, fuerza-trabajo-energía; calor-temperatura,...; casi el 60\% de los participantes. Tenemos la impresión que no cuidan el lenguaje en la comunicación (y no sólo en Ciencias...).

- Sabían lo que es ahorro energético, pero utilizaban argumentaciones pobres para justificar sus respuestas. Asociaban su importancia a cuestiones económicas y, en menor medida, al deterioro del medio ambiente.

- Usaban la idea de consumo energético "asociada" al ahorro energético. Algunos (casi una quinta parte) asociaban la clase $A$ al rendimiento, duración, precio... No señalaban las posibles variables de las que depende el consumo.

- Consideraban que la producción de energía es un problema solucionable: "se crean más centrales, parques, paneles... de energías alternativas, que no deterioran el medio ambiente".

- Parece que consideraban que los problemas energéticos son causados por "los demás". No planteaban medidas preventivas. Las medidas no parecían cambiar "sus" estilos de vida (sólo en la tercera parte se habla de acciones que les afectan directamente: principalmente relacionados con el trasporte o el uso de AV).

- Respecto a los conocimientos didácticos, los resultados son más inadecuados que los científicos.

- Casi todos (85/99) propusieron una actividad con un planteamiento similar al que hemos utilizado con ellos: visionado del spot elegido y preguntas sobre el mismo. No obstante, pocos (6/99) realizaron una breve introducción o señalaron alguna explicación previa antes de plantear la actividad.

- Parece que diseñaran una prueba de evaluación y no una actividad para enseñar un determinado contenido. En general, da la impresión que suponen que, con sólo preguntar, los alumnos van a responder adecuadamente -lo que demuestra que aprenden- sin más explicaciones ni consideraciones (¿aprendizaje por descubrimiento autónomo?)

- Encontramos problemas en la formulación de cuestiones: el número de preguntas resulta desproporcionado (48/99 plantean más de 8); utilizan -más de la mitad- un lenguaje inapropiado (términos confusos, frases largas, giros lingüísticos complejos...) para dirigirse a alumnos de Educación Primaria; muchas cuestiones -casi la tercera parte de las planteadas- no se apoyaban en el contenido del video, por lo que se podía haber prescindido de éste para hacer las preguntas que planteaban.

\section{En relación con el PP2}

Vamos a describir los resultados obtenidos en función de unas Unidades de Análisis: las subcompetencias implícitas.

\section{a) En relación con la identificación de ideas en AV (ítems 1, 4 y 6)}

Esta subcompetencia exige que el alumno sea capaz de localizar una información en el audio y/o la imagen del AV. Al respecto diremos que, en general, no tuvieron problemas con este tipo de preguntas. 
Así, más del $90 \%$ se refieren a la eficiencia energética y/o a la clase energética A en cuanto a lo que dice Casillas; y casi todos (97/98) reconocen los $21^{\circ} \mathrm{C}$ que Villa recomienda para las calefacciones. Más heterogeneidad hay en el ítem 4; aunque la mayor parte (81\%) describió que Cazorla hacía una seña al juez de línea para que apagara la pizarra (algunos hablan de "cartel"), el resto mencionó el acto deportivo de la sustitución, pero no establecieron ninguna relación con el ahorro energético.

Aunque minoritarios, los errores son llamativos porque introducían información que no contenía el anuncio (por ejemplo, hablaban del rendimiento, del medioambiente, de la contaminación...), pero estas afirmaciones van más allá de lo dicho y de lo visto.

\section{b) En relación con el significado de términos contenidos en el AV (ítems 2 y 8)}

Esta subcompetencia exige que el alumnado sepa explicar el significado de términos o expresiones contenidas en el AV. Obviamente el contenido del AV puede condicionar la respuesta, pero también se pueden apoyar en conocimientos propios, adquiridos en un contexto diferente. Preguntamos por "clase A" (ítem 2) y "termostato" (ítem 8).

Por una razón u otra los resultados han sido aceptables: el 81\% respondió adecuadamente la pregunta 2 y un $84 \%$ la pregunta 8 . Entre las no incluidas en estas categorías encontramos quienes asocian la clase $\mathrm{A}$ al rendimiento, a la duración del electrodoméstico o, incluso, al precio. Y, en el otro, la mayor parte de los errores se refieren a la confusión con un termómetro o con la medida de la Temperatura, pero también se deslizan confusiones terminológicas con el concepto de calor.

\section{c) En relación con las inferencias cercanas al contenido del AV (ítems 5, 7 y 11)}

Esta subcompetencia exige que el estudiante utilice la información contenida en el AV para responder la cuestión que se plantea. Probablemente por la naturaleza de este tipo de preguntas, los resultados son menos homogéneos que en las anteriores.

Por un lado, en las cuestiones 5 y 7 , casi todos -más del 95\%-centran su razonamiento en el ahorro energético. Es cierto que el nivel de la argumentación no es homogéneo y que, frente a los que detallaron sus justificaciones, otros respondieron sin alardes literarios.

Por otro, la cuestión 11 pone de manifiesto cómo existe una inferencia -o una trasferencia- de la propia actividad: los estudiantes "repiten", en gran medida, el modelo que hemos usado con ellos, sin tener presente que lo nuestro es un instrumento de evaluación y lo que les pedíamos era una actividad de enseñanza.

\section{d) En relación con el resumen de las ideas principales (ítem 9)}

Respecto a las tres ideas más importantes, los estudiantes plantearon 285 de las 297 posibles; hay que decir que algunos señalaron más de 3 .

La idea más señalada es "la importancia del ahorro energético" (más de la tercera parte de las respuestas); luego hay un conjunto de respuestas que se refieren al contenido de las cuatro situaciones (a la conveniencia de usar trasporte público, a la utilización de electrodomésticos de clase $\mathrm{A}$, a poner la calefacción a una temperatura adecuada y, en menor medida, a apagar los aparatos eléctricos).

Pero también hay referencias a ideas no contempladas implícitamente en el AV: la conservación del medio-ambiente, al consumo energético y, en menor medida, a la contaminación energética. Nuevamente lo que se sugiere se superpone a lo que se dice. 


\section{e) En relación con el posicionamiento argumentado (ítems 3 y 9)}

Esta subcompetencia exige que el alumnado manifieste sus creencias u opiniones de forma razonada ante algo que se dice o se ve en el AV.

En nuestro caso, en el ítem 3, debían explicar la importancia de usar el trasporte público y algunos utilizaron varios argumentos. Las ideas más repetidas se refieren a "que se ahorra energía" (75\%); "que el consumo de gasóleo, gasolina, petróleo... es menor que si cada uno usa su coche" (30\%); incluso, algunos (12\%) "que se deberían utilizar bicicletas". Otros hicieron afirmaciones no relacionadas con el ahorro energético (comodidad en el desplazamiento, seguridad...).

Nos ha llamado la atención que algunos (7\%), debido a una situación problemática del momento, se refirieran al: derecho al trasporte de los alumnos, problemas derivados del distrito único... Valoramos muy positivamente el interés del futuro maestro por los problemas del contexto.

En el ítem 9, un $80 \%$ se limitaron a elegir una de las opciones, pero sin justificarlas; en los demás, simplemente no contestan o dicen que no lo saben. Se pone de manifiesto el desconocimiento sobre el tema. Es cierto que, cuando formulamos la pregunta, a diferencia de otras, no les pedimos que lo justificaran, pero parecía obvio en el desarrollo de la prueba. Creemos que las carencias (identificación de problemas, estrategias de resolución, los datos y establecimiento de conclusiones) están detrás de estos resultados.

\section{f) En relación con la búsqueda de información a partir de AV}

Esta subcompetencia exige al estudiante conocer estrategias para buscar información (no necesariamente en internet) sobre datos, resultados, significados, conocimientos... sobre la temática que aborda el AV. En nuestro cuestionario, está el ítem 14.

Como en la anterior, las respuestas nos aportaron mucha información (quizás, habría que plantearla de otra manera). A pesar de la escasa información recogida (más de la mitad la dejaron en blanco), podemos decir que predominaban dos estrategias: buscar directamente en un buscador y acudir a algunas direcciones (las más conocidas eran Wikipedia, Rincón del Vago...). En ningún caso, eligieron una específica de ciencias.

\section{Conclusiones}

Obviamente, en un trabajo de esta naturaleza, no se pueden generalizar los resultados ni extraer conclusiones concluyentes (habría que ampliar el número de participantes y, por supuesto, plantear otras situaciones en las que estuvieran presentes estas y otras subcompetencias). No obstante, en nuestro estudio, podemos decir:

- que no se ha detectado una mejora en los conocimientos científicos con los que llega el estudiante, a pesar de la LOE, de la LOMCE...

- que es preciso revisar los contenidos de las materias psicopedagógicas que imparten en los primeros cursos de la titulación. Nuestros resultados ponen de manifiesto que el uso de etiquetas y afirmaciones "políticamente correctas" no llevan consigo una concreción en las actividades planteadas por los futuros maestros.

- que los resultados han sido satisfactorios en la identificación de ideas en un AV, en el significado de términos, en algunas inferencias cercanas (curiosamente las no didácticas) e, incluso, en la de posicionamiento argumentado. Pero que, en las restantes, los resultados no han sido los esperados, lo que requiere una formación de nuestros estudiantes porque "el que no sabe, no sabe enseñar". 
Por último, creemos que, a menudo, existe una queja bastante extendida de que nuestros alumnos llegan con grandes carencias en sus conocimientos científicos. En esta experiencia hemos encontrado que también existen lagunas importantes desde el punto de vista psicopedagógico y éstas también hay que tenerlas presentes en nuestros programas formativos de Didáctica de las Ciencias Experimentales.

\section{Referencias bibliográficas}

Bravo, P. y Cofré, H. (2016) Developing biology teachers pedagogical content knowledge through learning study: the case of teaching human evolution. International Journal of Science Education, 38(16), 2500-2527. DOI: https://doi.org/10.1080/09500693. 2016.1249983

Gairín, J. (coord.) (2009). La enseñanza de las ciencias en el sistema educativo panameño. Evaluación del profesorado. Ciudad de Panamá. Ministerio de Educación (República de Panamá) y Senacyt.

Grangeat, M. y Kapelari, S. (2015) Introduction: Exploring the growth of science teacher's professional knowledge. En M. Grangeat (Ed.), Understanding science teacher's professal knowledge growth (pp. 1-12). Rotterdam, The Netherlands: Sense Publishers.

Jarvis, T. y Pell, A. (2004). Primary teachers' changing attitudes and cognition during a twoyear science in-service programme and their effect on pupils. International Journal of Science Education, 26(14), 1787-1811. DOI: https://doi.org/10.1080/095006904 2000243763

Jiménez, M.P. (2010). 10 Ideas Clave. Competencias en argumentación y uso de pruebas. Barcelona: Graó.

Marcelo, C. (2011). Evaluación del desarrollo profesional docente. Barcelona: Díada

Mellado, V. (2001). ¿Por qué a los profesores de ciencias nos cuesta tanto cambiar nuestras concepciones y modelos didácticos? Revista Interuniversitaria de Formación del Profesorado, 40, 17-30. Recuperado de: https://www.aufop.com/aufop/uploaded_ files/articulos/1223575368.pdf

Opre, D. (2015) Teachers' conceptions of assessment. Procedia-Social and Behavioral Sciences, 209, 229-233. DOI: https://doi.org/10.1016/j.sbspro.2015.11.222

Pedrinaci, E. (coord.) (2012). 11 ideas clave. El desarrollo de la competencia científica. Barcelona: Graó.

Perales, F. J. (2018). El área de Didáctica de las ciencias Experimentales en España: entre la tribulación y la esperanza. Ápice. Revista de Educación Científica, 2(2), 1-14. DOI: https://doi.org/10.17979/arec.2018.2.2.3915

Pérez Esteve, P. (2009). La comprensión lectora y la competencia en comunicación lingüística en el nuevo marco curricular: algunas claves para su desarrollo. Educatio Siglo XXI, 27 (1), 13-32. Recuperado de: http://revistas.um.es/educatio/article/ view/71081/68621

Pro, A. (2014). La energía: uso, consumo y ahorro energético en la vida cotidiana. Barcelona: Graó.

Pro, A. y Saura, O. (2000). ¿Qué contenidos conceptuales utilizan los profesores cuando planifican unidades didácticas en la educación secundaria? Alambique, 24, 87-98. 
Pro, A. y Saura, O. (2007). La planificación: un proceso para la formación, la innovación y la investigación. Alambique, 52, 39-55.

Pro, A., Saura, O. y Sánchez, G. (2000). ¿Qué actividades de enseñanza utilizan los profesores en formación inicial y los profesores en ejercicio cuando planifican unidades didácticas? Investigación en la Escuela, 40, 23-37. Recuperado de: http:// www.investigacionenlaescuela.es/articulos/40/R40_2.pdf

Pro, C. y Pro, A. (2012). ¿Cómo utilizan sus conocimientos maestros en formación inicial cuando realizan una actividad de laboratorio? En L. del Río e I.Teva, FEICES 2012 (pp. 1315-1321). Santiago: USC.

Pro, C., Pro, A. y Serrano, F.J. (2017). ¿Saben los maestros en formación inicial qué subcompetencias están trabajando cuando diseñan una actividad de enseñanza? Enseñanza de las Ciencias, 35(3), 7-28. DOI: https://doi.org/10.5565/rev/ ensciencias. 2205

Pro, C., Pro, A. y Serrano, F.J. (2018). ¿Cómo utilizan los maestros en formación inicial sus conocimientos didácticos en el diseño de una prueba experiencial para evaluar subcompetencias de estudiantes de Educación Primaria? Enseñanza de las Ciencias, 36 (2), 43-62. DOI: https://doi.org/10.5565/rev/ensciencias.2498

van Driel, J. H., Beijaard, D. y Verloop, N. (2001). Professional development and reform in science education: The role of teachers' practical knowledge. Journal of Research en Science Teaching, 38(2), 137-158. DOI: https://doi.org/10.1002/10982736(200102)38:2\%3C137::AID-TEA1001\%3E3.0.CO;2-U 
